\title{
CHERN CLASSES OF CERTAIN REPRESENTATIONS OF SYMMETRIC GROUPS
}

BY

\author{
LEONARD EVENS AND DANIEL S. KAHN ${ }^{1}$
}

\begin{abstract}
A formula is derived for the Chern classes of the representation id $\int \xi: P \int H \rightarrow U_{p n}$ where $P$ is cyclic of order $P$ and $\xi: H \rightarrow U_{n}$ is a fintie dimensional unitary representation of the group $H$. The formula is applied to the problem of calculating the Chern classes of the "natural" representations $\pi_{j}: \delta_{j} \rightarrow U_{j}$ of symmetric groups by permutation matrices.
\end{abstract}

1. Introduction. In [Ch], one of us derived "formulas" for the Chern classes of an induced representation in the case where the inducing representation of the subgroup is 1-dimensional. The formula for the $i$ th Chern class involved a leading term expressed in terms of the multiplicative generalization of transfer in [N] plus additional terms arising from Chern classes $c_{j}\left(\pi_{k}\right)$ of "natural representations" by permutation matrices. The situation 'was 'somewhat unsatisfactory since little or nothing was known about the classes $c_{j}\left(\pi_{k}\right)$.

Recently, through access to an interesting paper of C. B. Thomas [Th], our attention was drawn to this question again. Thomas makes use of estimates of Grothendieck [G] on the orders of Chern classes of representations of discrete groups. Grothendieck's results [G, Corollary 4.11, p. 263] imply that, for a rational representation $\rho: G \rightarrow \mathrm{GL}(n, Q)$, the $p$-primary component of the order of $c_{i}(\rho)$ is bounded by

$$
\begin{cases}p^{0} & \text { if } i \neq 0 \bmod p-1, \\ p^{1+p_{p}(i)} & \text { if } i \equiv 0 \bmod p-1,\end{cases}
$$

for $p$ odd, and

$$
\begin{cases}2^{1} & \text { if } i \text { is odd, } \\ 2^{2+\nu_{2}(i)} & \text { if } i \text { is even, }\end{cases}
$$

for $p=2$.

Here, $i=p^{\nu_{p}(i)} i^{\prime}$ where $\left(i^{\prime}, p\right)=1$. (See [Th, §2], for detailed derivation.) In

Received by the editors April 28, 1976.

AMS (MOS) subject classifications (1970). Primary 20C00, 55F40; Secondary 18H10, $20 \mathrm{C} 30$.

Key words and phrases. Chern classes, group, symmetric groups, wreath products, induced representation, transfer, double complex.

'The research reported on in this paper was partially supported by NSF Grants MPS71-02912 A05 (first author) and MPS75-06976 (second author). 
this paper, we show for $\rho=\pi_{p^{m}}: \delta_{p^{m}} \rightarrow \mathrm{GL}\left(p^{m}, \mathrm{Z}\right)$, the $p$-primary component is exactly the Grothendieck bound, for $p$ odd, but one-half that bound for $p=2, i$ even, $\left(i<p^{m}\right)$. In the process, we independently establish Grothendieck's bounds for the representations $\pi_{k}$ without use of étale cohomology. (The anomalous situation for $p=2$ may have something to do with rational representations of Quaternion groups.)

In order to calculate these orders, we needed the first steps in the analysis of representations induced from representations (of a subgroup) of degree greater than one. Although the method is quite general, it yields the most intelligible result for normal subgroups of prime index ( $\$ 2$, Theorem III). This result and its analogues, for many readers, may be of greater interest than the results on orders of Chern classes discussed above.

2. Chern classes of an induced representation of prime index. Let $H$ be a subgroup of the group $G$, and let $\xi: H \rightarrow U_{n}$ be a finite dimensional unitary representation of $H$. In [Ch, Theorem 4, p. 190], there is a formula for the Chern classes of the induced representation $\rho=\operatorname{ind}_{H \rightarrow G} \xi$ in the special case $\xi$ is 1-dimensional $(n=1)$. Here we derive a parallel formula for general $n$ but in the special case $H$ is normal and $(G: H)=p$ is prime. The methods are similar to those in [Ch] and [N], and we use the terminology and results of those papers freely.

As in [Ch, p. 189, 2nd paragraph of Remark], the induced representation may be dissected by wreath products:

$$
G \stackrel{\Phi}{\rightarrow} P \int H^{\mathrm{id}} \stackrel{\mathcal{S} \xi}{\rightarrow} P \int U_{n} \longrightarrow U_{p n} .
$$

Here $\Phi$ is the "Frobenius" imbedding of $G$ in $\delta_{p} \int H$. (See $[\mathbf{N}, \S 2$, pp. 54-55].)

Because of the hypotheses on $H$, we may replace $\delta_{p}$ by its $p$-Sylow subgroup $P$ which we may take to be generated by the cycle $\sigma=(123 \ldots p)$. Note also that, in the last inclusion, $P \cong P \int 1$ is imbedded in $U_{p n}$ as "block" permutation matrices. One useful way of visualizing this is as follows: First imbed $P$ in $\delta_{p n}$ by

$$
\begin{aligned}
\sigma= & (1,2, \ldots, p) \rightarrow(1, n+1, \ldots,(p-1) n+1) \\
& \cdot(2, n+2, \ldots,(p-1) n+2) \ldots(n, 2 n, \ldots, p n)
\end{aligned}
$$

and then imbed $\delta_{p n}$ in $U_{p n}$ by its natural representation $\pi_{p n}$ as permutation matrices. Alternately, the imbedding of $P$ in $\delta_{p n}$ may be obtained by 


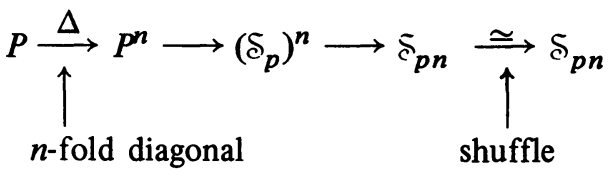

[The last "shuffle" is the inner automorphism of $\delta_{p n}$ arising from the appropriate rearrangement of $\{1,2, \ldots, p n\}$.] We shall make use of these descriptions below.

The crucial step in our argument is the calculation of the Chern classes of the inclusion representation $\iota_{n}: P \int U_{n}<U_{p n}$. Since $H^{*}\left(B U_{n}, \mathrm{Z}\right)$ is Z-free, the argument of Nakaoka in [Ch, pp. 181-182] shows that

$$
H^{*}\left(B\left(P \int U_{n}\right), \mathrm{Z}\right) \cong H^{*}\left(P, H^{*}\left(B U_{n}, \mathrm{Z}\right)^{p}\right)
$$

as graded rings. Moreover, the isomorphism is consistent with all the homomorphisms (such as restriction and transfer) we shall have need of. (See [Ch, pp. 181-182].) We shall need some notation. Let $c=1+c_{1}+c_{2}+\cdots+c_{n}$ be the total Chern class in $H^{*}\left(B U_{n}\right) \cong \mathbf{Z}\left[c_{1}, c_{2}, \ldots, c_{n}\right]$, and let $\mu$ be a generator of $H^{2}(P, Z)$. (It does not matter which generator is used because only $\mu^{p-1}$ appears in our formulas.)

THEOREM I. With the notation as above, the total Chern class of $\iota_{n}$ is given by

$$
\begin{aligned}
c\left(\iota_{n}\right)= & c \times c \times \cdots \times c+\left[\left(1-\mu^{p-1}\right)^{n}-1\right] \\
& +\left[\left(1-\mu^{p-1}\right)^{n-1}-1\right]\left(c_{1} \times c_{1} \times \cdots \times c_{1}\right) \\
& +\left[\left(1-\mu^{p-1}\right)^{n-2}-1\right]\left(c_{2} \times c_{2} \times \cdots \times c_{2}\right) \\
& +\ldots \\
& +\left(-\mu^{p-1}\right)\left(c_{n-1} \times c_{n-1} \times \cdots \times c_{n-1}\right)
\end{aligned}
$$

where each iterated cross product $\left[\right.$ in $\left.\left(H^{*}\left(B U_{n}\right)^{p}\right)^{P}\right]$ is p-fold.

Explication. For $p=3, n=3$, the bidegrees with nonzero contributions are indicated below. (The true degrees are twice those indicated.)

Thus, on the vertical edge appear the Chern classes of the inclusion of $\left(U_{n}\right)^{p}$ in $U_{p n}$, i.e. the components of $c \times c \times \cdots \times c$. (These are, of course, invariant under $P$.) The additional contributions along the horizontal rows, $H^{*}\left(P, H^{2 s}\left(\left(B U_{n}^{p}\right), \mathrm{Z}\right)\right), 0<s<p n$, are zero unless $s$ is divisible by $p$. If so, $s=p t$, and the contribution along that row is essentially

$$
\left(1-\mu^{p-1}\right)^{n-t}(\underbrace{c_{t} \times \cdots \times c_{t}}_{p \text {-times }}) \text {. }
$$




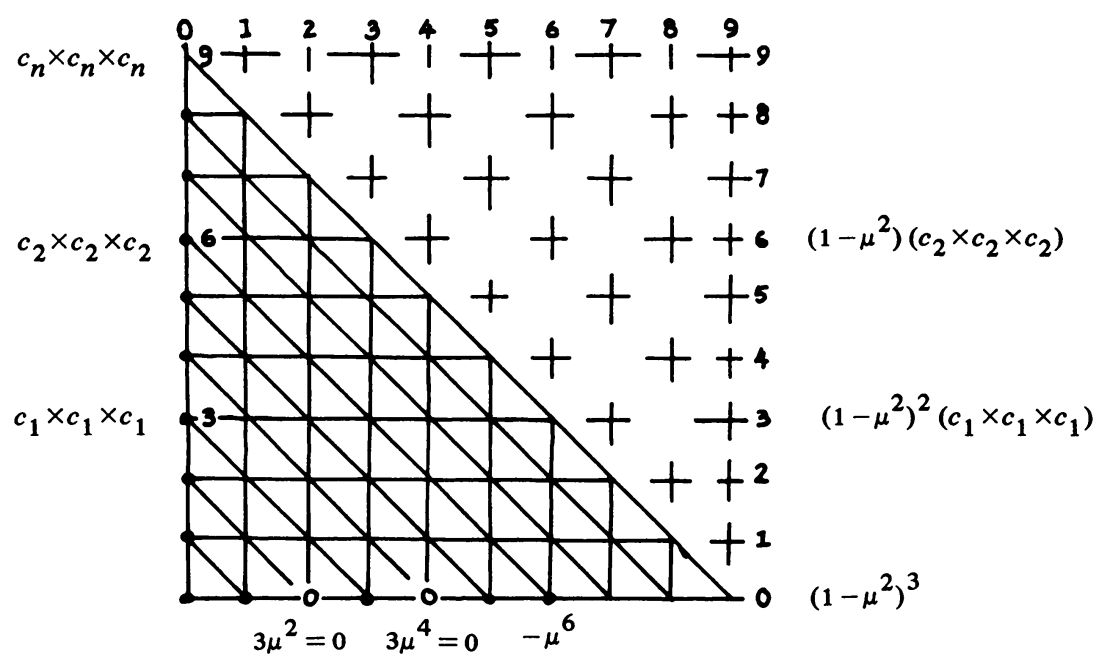

We use $\left(1-\mu^{p-1}\right)^{n-t}-1$ in the formula in the theorem to avoid counting $c_{t} \times c_{t} \times \cdots \times c_{t}$ twice. $1-\mu^{p-1}$ is, of course, the total Chern class $c\left(\pi_{p}\right)$ of the natural representation ( $=$ regular representation) of $P$ in $U_{p}$. Its powers are nonzero only in degrees $\equiv 0$ mod $2(p-1)$. Finally, we remark that, in formula (B), $c_{t} \times c_{t} \times \cdots \times c_{t}$ may be replaced by $(c \times c \times \cdots \times c)_{p t}$, the component of the $c \times c \times \cdots \times c$ of degree $2 s=2 p t$. (That will be apparent in the proof.)

Proof of Theorem. Consider $H^{*}\left(B U_{n}^{p}, \mathbf{Z}\right) \cong \mathbf{Z}\left[c_{1}, c_{2}, \ldots, c_{n}\right]^{p}$ as a module over $P$. It decomposes into a direct sum of homogeneous submodules, each of which is isomorphic either (i) to $Z$ or (ii) to $Z[P]$. The first type are generated over $P$ by monomials of the form $c_{i} \times c_{i} \times \cdots \times c_{i}$ (or products of such) and the second type are generated over $P$ by monomials which necessarily involve factors of the form $c_{i_{1}} \times c_{i_{2}} \times \cdots \times c_{i_{p}}$ where at least two indices differ. In particular, $H^{r}\left(P, H^{2 s}\left(B U_{n}^{p}\right)\right)=0$ for $r>0$ when $s \not \equiv 0$ $\bmod p$. (Only type (ii) submodules $\mathbf{Z}[p]$ occur in that case.) Accordingly, there are no nonzero contributions to the Chern classes along (the positive part of) those rows $(s \not \neq 0 \bmod p$.)

$U_{n}$ contains a maximal torus $T^{n}$ so that $P \int U_{n}$ contains the subgroup $P \int\left(T^{n}\right) \cong P \int_{p}\left(1 \int_{n} T\right) \cong\left(P \int_{p} 1\right) \int_{p n} T$. (The subscript indicates the permutation degree and also the number of factors of the following subgroup to take.) We shall show res: $H^{*}\left(B\left(P \int U_{n}\right), \mathrm{Z}\right) \rightarrow H^{*}\left(B\left(\left(P \int_{p} 1\right) \int_{p n} T\right), \mathrm{Z}\right)$ is a monomorphism. (Then the methods of [Ch] may be used to calculate the Chern classes.) First, recall that $H^{*}\left(B U_{n}, \mathbf{Z}\right)=\mathbf{Z}\left[c_{1}, c_{2}, \ldots, c_{n}\right]$ restricts injectively onto a direct summand of $H^{*}\left(B T^{n}, \mathbf{Z}\right)=\mathbf{Z}\left[x_{1}, x_{2}, \ldots, x_{n}\right], c_{i}$ going onto the $i$ th elementary symmetric function in $x_{1}, x_{2}, \ldots, x_{n}$. (The splitting may be checked directly by appropriate change of basis; see also [Bo, $\$ 20$, p. 66] for a more general result along these lines.) It follows that $H^{*}\left(\left(B U_{n}\right)^{p}\right) \cong$ 
$\mathbf{Z}\left[c_{1}, \ldots, c_{n}\right]^{p}$ is a direct summand of $H^{*}\left(\left(B T^{n}\right)^{p}\right) \cong \mathbf{Z}\left[x_{1}, \ldots, x_{n}\right]^{p}$ even as $P$-module. (Recall $P$ acts by permuting the factors.) Hence,

$$
H^{*}\left(P, H^{*}\left(B U_{n}\right)^{p}\right) \rightarrow H^{*}\left(P, H^{*}\left(B T^{n}\right)^{p}\right)
$$

is a monomorphism. Now apply the Nakaoka isomorphisms. [Ch, pp. 181-182.]

To analyze the situation in $\left(P \int 1\right) \int T$, we resort to the diagram in Figure 1 .

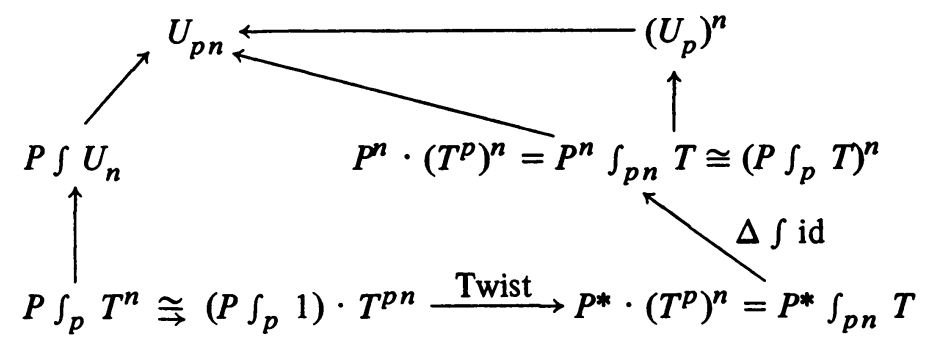

FIGURE 1

Here $P^{*}$ is just $P$ again but, as permutation group, its action is obtained as follows: $P$ acts on $T^{p}$ (as usual), hence $P^{n}$ acts on $\left(T^{p}\right)^{n}$; let $P^{*}$ act by composing with the diagonal $\Delta: P^{*} \rightarrow P^{n}$. We may now make the desired calculation by descending the diagram on the right. First, however, we need some notation. Viewing $\left(T^{n}\right)^{p}$ as a subgroup of $\left(U_{n}\right)^{p}$ (on the left), let $H^{*}\left(\left(B T^{n}\right)^{p}, \mathbf{Z}\right)=\mathbf{Z}[X]$ where

$$
\begin{aligned}
X=\left\{x_{1}, x_{2}, \ldots, x_{n},\right. & \\
& x_{n+1}, x_{n+2}, \ldots, x_{2 n}, \\
& \vdots \\
& \left.x_{(p-1) n+1}, x_{(p-1) n+2}, \ldots, x_{p n}\right\} .
\end{aligned}
$$

The block structure indicates the segregation of the factors. $P$ permutes the $p$ rows cyclically. On the right, let $H^{*}\left(B T^{p}, \mathbf{Z}\right)=\mathbf{Z}\left[y_{1}, y_{2}, \ldots, y_{p}\right]$ where $y_{1}=x_{1}, y_{2}=x_{n+1}, \ldots, y_{p}=x_{(p-1) n+1}$, and let $H^{*}\left(\left(B T^{p}\right)^{n}, \mathbf{Z}\right)=\mathbf{Z}[Y]$ where $Y$ is identical with $X$, but with elements renamed $y_{1}, \ldots, y_{p n}$ and reordered by successively traversing the columns in the above presentation of $X$.

$$
\begin{gathered}
Y=\left\{y_{1}, y_{p+1}, \ldots, y_{(n-1) p+1}\right. \\
y_{2}, y_{p+2}, \ldots, y_{(n-1) p+2} \\
\vdots \\
\left.y_{p}, y_{2 p}, \ldots, y_{n p}\right\}=X .
\end{gathered}
$$

$P$ acts cyclically on each column of $y^{\prime}$; hence $P^{n}$ and $P^{*}(=P)$ act on $Y$ as 
before. Let $\phi_{1}, \phi_{2}, \ldots, \phi_{p}$ be the elementary symmetric functions of $y_{1}$, $y_{2}, \ldots, y_{p}$. Write $\dot{\phi}=\phi_{1}+\cdots+\phi_{p}$. More generally, write $\dot{\phi}^{(1)}=\dot{\phi}$, and let $\dot{\phi}^{(2)}=\phi_{1}^{(2)}+\cdots+\phi_{p}^{(2)}, \ldots, \dot{\phi}^{(n)}$ have corresponding meanings for the other blocks of $Y$. Finally, we shall have occasion to use cross-product notation so that subject to appropriate identifications, $1 \times \dot{\phi} \times \cdots \times 1=$ $\dot{\phi}^{(2)}$, etc.

LEMMA. In $H^{*}\left(B\left(P \int_{p} T\right), \mathrm{Z}\right) \cong H^{*}\left(P, H^{*}(T)^{p}\right)$, the total Chern class is given by

$$
c\left(\iota_{1}\right)=\dot{\phi}+1-\mu^{p-1} .
$$

Proof of Lemma. This result is contained in [Ch, Corollary 5, p. 190] but the order of presentation there does not make this clear. However, in view of the discussion of the $\boldsymbol{P}$-module structure of $\mathbf{Z}\left[y_{1}, \ldots, y_{p}\right]$ above, the result follows easily from the following diagram: (true degree $=$ twice indicated degree.)

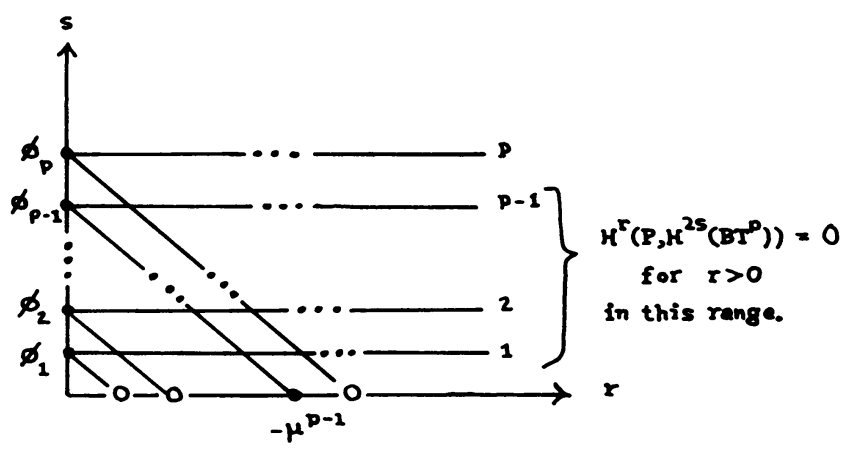

FIGURE 2

That $\dot{\phi}$ and $1-\mu^{p-1}=c\left(\pi_{p}\right)$ appear on the edges is fairly clear, and it is discussed in [Ch, \$3, pp. 183-184].

To continue the proof of the theorem, in $H^{*}\left(B\left(P \int T\right)^{n}\right)$,

$$
\begin{aligned}
c\left(\iota_{1} \times\right. & \left.\cdots \times \iota_{1}\right)=c\left(\iota_{1}\right) \times c\left(\iota_{1}\right) \times \cdots \times c\left(\iota_{1}\right) \\
& =\left(\dot{\phi}+1-\mu^{p-1}\right) \times\left(\dot{\phi}+1-\mu^{p-1}\right) \times \cdots \times\left(\dot{\phi}+1-\mu^{p-1}\right) .
\end{aligned}
$$

The descent to $P^{*} \cdot T^{p n}$ requires a description of the homomorphism induced by

$$
\Delta \int \text { id: } P^{*} T^{p n}=P^{*} \int_{p n} T \rightarrow P^{n} \int_{p n} T \stackrel{\widetilde{\simeq}}{\rightarrow}\left(P \int_{p} T\right)^{n} .
$$

Making use of the Nakaoka isomorphisms, we need

$$
\begin{aligned}
H^{*}\left(P^{n}, \mathbf{Z}\left[y_{1}, \ldots, y_{p}\right]^{n}\right) \cong H^{*}\left(P, \mathbf{Z}\left[y_{1}, \ldots, y_{p}\right]\right)^{n} & H^{*}\left(P^{*}, \mathbf{Z}\left[y_{1}, \ldots, y_{p}, y_{p+1}, \ldots, y_{p n}\right]\right) .
\end{aligned}
$$


Because of the naturality of the Nakaoka isomorphisms with respect to change of permutation group, this homomorphism is essentially just the $\boldsymbol{n}$-fold cup product for the cohomology of $\boldsymbol{P}$ based on the $\boldsymbol{n}$-fold "pairing" $\mathbf{Z}\left[y_{1}, \ldots, y_{p}\right]^{n} \stackrel{\simeq}{\rightarrow} \mathbf{Z}\left[y_{1}, y_{2}, \ldots, y_{p}, \ldots, y_{p n}\right]$. Thus, the desired Chern class at the bottom level is the product

$$
\left(\dot{\phi}^{(1)}+1-\mu^{p-1}\right) \cup\left(\dot{\phi}^{(2)}+1-\mu^{p-1}\right) \cup \cdots \cup\left(\dot{\phi}^{(n)}+1-\mu^{p-1}\right) .
$$

To complete the proof, we must expand this product and show it agrees with the expression in the theorem. If we expand in powers of $1-\mu^{p-1}$, a typical term is

$$
\left(\sum_{1<i_{1}<i_{2}<\cdots<i_{t}<n} \dot{\phi}^{\left(i_{1}\right)} \dot{\phi}^{\left(i_{2}\right)} \cdots \dot{\phi}^{\left(i_{t}\right)}\right)\left(1-\mu^{p-1}\right)^{n-t},
$$

$0<t<n$. Call the sum in parentheses $\psi_{t}$. Then it will be convenient to rewrite $(\mathrm{C})$ as

$$
\psi_{t}\left(1-\mu^{p-1}\right)^{n-t}=\psi_{t}+\psi_{t}\left[\left(1-\mu^{p-1}\right)^{n-t}-1\right] .
$$

Assume $t>0\left(\psi_{t} \neq 1\right)$ and $t<n\left(\left(1-\mu^{p-1}\right)^{n-t} \neq 1\right)$. We claim that most of the terms $\psi_{t}$ are annihilated by $\left(1-\mu^{p-1}\right)^{n-t}-1$. For, by our previous analysis, viewing $\mathbf{Z}\left[y_{1}, y_{2}, \ldots, y_{p n}\right]$ as a $P^{*}$-module, we need only include terms contained in factors isomorphic to $\mathbf{Z}$ and we can exclude terms arising entirely from factors isomorphic to $Z[P]$, since products of such with $[(1-$ $\left.\left.\mu^{p-1}\right)^{n-t}-1\right]$ are zero. Hence we can exclude

$$
\begin{aligned}
& \phi_{1}=y_{1}+y_{2}+\cdots+y_{p}, \\
& \phi_{2}=\left(y_{1} y_{2}+y_{2} y_{3}+\cdots+y_{p-1} y_{p}\right)+\left(y_{1} y_{3}+y_{2} y_{4}+\cdots\right)+\cdots
\end{aligned}
$$

(which involves $(p-1) / 2$ factors isomorphic to $Z[P]), \phi_{3}, \ldots, \phi_{p-1}$. Thus, we can rewrite (D) as

$$
\begin{aligned}
\psi_{t}\left(1-\mu^{p-1}\right)^{n-t}= & \psi_{t}+\left(\sum_{1<i_{1}<\cdots<i_{t}<n} \phi_{p}^{\left(i_{1}\right)} \cdots \phi_{p}^{\left(i_{i}\right)}\right) \\
& \cdot\left[\left(1-\mu^{p-1}\right)^{n-t}-1\right] .
\end{aligned}
$$

The sum in parentheses is of degree $t p$ (in the variables $Y$ ), and is part of the $t p$ th elementary symmetric function in the $n p$ variables $y_{1}, y_{2}, \ldots, y_{n p}$. (Call that $\Phi_{t p}$.)

The additional monomials in $\Phi_{t p}$ generate direct summands isomorphic to $Z[P]$. (All monomials left invariant by $P^{*}$ are already in the above sum.) Hence, these monomials may be added without affecting the product and we obtain

$$
\psi_{t}\left(1-\mu^{p-1}\right)^{n-t}=\psi_{t}+\Phi_{t p}\left[\left(1-\mu^{p-1}\right)^{n-t}-1\right]
$$


To get the total Chern class, add up all the above terms (including $t=0$ and $t=n)$. The result is

$$
\begin{aligned}
1+\sum_{t=1}^{n-1} \psi_{t} & +\psi_{n}+\left[\left(1-\mu^{p-1}\right)^{n}-1\right] \\
& +\sum_{t=1}^{n-1} \Phi_{t p}\left[\left(1-\mu^{p-1}\right)^{n-t}-1\right] .
\end{aligned}
$$

On the other hand, the first three terms,

$$
\begin{aligned}
1+\sum_{t=1}^{n-1}\left(\sum_{1<i_{1}<}\right. & \left.\sum_{<i_{t}<n} \dot{\phi}^{\left(i_{1}\right)} \cdots \dot{\phi}^{\left(i_{n}\right)}\right)+\dot{\phi}^{(1)} \dot{\phi}^{(2)} \ldots \dot{\phi}^{(n)} \\
& =\left(1+\dot{\phi}^{(1)}\right)\left(1+\dot{\phi}^{(2)}\right) \cdots\left(1+\dot{\phi}^{(n)}\right) \\
& =1+\Phi_{1}+\Phi_{2}+\cdots+\Phi_{p n}=\Phi,
\end{aligned}
$$

where $\Phi_{i}$ is the $i$ th elementary symmetric function in $y_{1}, y_{2}, \ldots, y_{p n}$. Hence, we obtain finally

$$
\Phi+\sum_{t=0}^{n-1} \Phi_{t p}\left[\left(1-\mu^{p-1}\right)^{n-t}-1\right] .
$$

$\Phi$, of course, is the total Chern class of the torus $\left(T^{p}\right)^{n}=\left(T^{n}\right)^{p}$. Following Figure 1 down on the left, we carry $c \times c \times \cdots \times c$ to $\Phi$; hence we have accounted for the term $c \times c \times \cdots \times c$ in the basic formula. To complete the proof, now call the variables $x_{1}, x_{2}, \ldots, x_{p n}$. (Technically, this involves a reordering and some twisting, but the $\Phi_{i}$ are clearly unchanged.) Note that, by means of the $P$-module argument, we can replace $\Phi_{t p}$ by any sub-sum which is $\boldsymbol{P}$-invariant and which contains all $\boldsymbol{P}$-invariant monomials of degree $p t$ : (e.g. $x_{1} x_{n+1} x_{2 n+1} \cdots x_{(p-1) n+1}=y_{1} y_{2} \cdots y_{p}=\phi_{p}^{(1)}$, generally $\left.\phi_{p}^{\left(i_{1}\right)} \phi_{p}^{\left(i_{2}\right)} \cdots \phi_{p}^{\left(i_{1}\right)}\right)$. Let $\tilde{\phi}_{i}$ be the $i$ th elementary symmetric function of the $n$ variables $x_{1}, x_{2}, \ldots, x_{n}$. Then one such sub-sum is

$$
\underbrace{\tilde{\phi}_{t} \times \tilde{\phi}_{t} \times \cdots \times \tilde{\phi}_{t}}_{p \text {-terms }} \text {. }
$$

Since this last expression is the image of $c_{t} \times c_{t} \times \cdots \times c_{t}$, we have completed the proof of the theorem.

To continue our analysis, suppose as before that $\xi: H \rightarrow U_{n}$ is a unitary representation of $\boldsymbol{H}$. We wish to find the Chern classes of id $\int \xi: P \int H \rightarrow$ $P \int U_{n}<U_{p n}$, and to do so we simply carry the results of Theorem I back to $P \int \boldsymbol{H}$ by (id $\left.\int \boldsymbol{\xi}\right)$.

In general, given $\alpha \in H^{*}(B H, Z)$ involving only even degrees, we can define a class $1 \int \alpha \in H^{*}\left(B\left(P \int H\right), Z\right)$. (See [N, \$4, pp. 56-58] for definition and properties.) If $\alpha$ is of degree $2 i, 1 \int \alpha$ is of degree 2ip. Moreover, for $H=U_{n}$, 
we have the Nakaoka isomorphism

$$
H^{*}\left(B\left(P \int U_{n}\right), \mathrm{Z}\right) \cong H^{*}\left(P, H^{*}\left(B U_{n}\right)^{p}\right)
$$

and $1 \int \alpha$ corresponds to $\alpha \times \alpha \times \cdots \times \alpha \in H^{0}\left(P, H^{*}\left(B U_{n}\right)^{p}\right)$. (This follows directly from the definition and the formal properties of the Nakaoka isomorphism.) [Note: If $H^{*}(H, \mathrm{Z})$ is not $\mathrm{Z}$-free, not only does the Nakaoka argument fail, but the spectral sequence $H^{*}\left(P, H^{*}\left(H^{p}, \mathrm{Z}\right)\right) \Rightarrow H^{*}\left(P \int H, \mathrm{Z}\right)$ behaves badly. $E_{2} \neq E_{\infty}$, and as we shall see below, the filtration in $H^{*}\left(P \int H, \mathrm{Z}\right)$ need not split. All this should be distinguished from the original case investigated by Nakaoka for coefficient ring $Z / p Z$. This is one example of several in which the theory for coefficients in $\mathbf{Z}$ differs radically from that for coefficients in a field.]

Because of the naturality of the wreath product construction, we have

$$
\left(\text { id } \int \xi\right)^{*}(c \times c \times \cdots \times c)=\left(\operatorname{id} \int \xi\right)^{*}\left(1 \int c\right)=1 \int \xi^{*}(c)
$$

and similar formulas with $c_{i}$ replacing $c$. Also (id $\left.\int \xi\right)^{*}(\mu)=\mu$. Hence, the translation of Theorem I to $P \int H$ reads as follows.

THEOREM II. Let $\xi: H \rightarrow U_{n}$ be a unitary representation of $H$, and let $P$ be a cyclic permutation group of prime order $p$ and degree $p$. Let $\mu$ generate $H^{2}(P, \mathbf{Z})$. Then

$$
\begin{aligned}
c\left(\text { id } \int \xi\right)= & 1 \int c(\xi)+\sum_{t=1}^{n-1}\left(1 \int c_{t}(\xi)\right)\left[\left(1-\mu^{p-1}\right)^{n-t}-1\right] \\
& +\left[\left(1-\mu^{p-1}\right)^{n}-1\right] .
\end{aligned}
$$

The last step is to carry Theorem II back to $G$ in the case $H$ is a normal subgroup of $G$ of index $p$. This requires composing with $\Phi^{*}$ where $\Phi$ is the Frobenius imbedding discussed earlier. Generally, we define $\Re_{H \rightarrow G}(\alpha)=$ $\Phi^{*}\left(1 \int \alpha\right)$. (See [N, $\$ \S 5$ and 6, pp. 58-62] for definition and properties.) As above, if $\alpha \in H^{2 i}(H, Z), \mathscr{T}(\alpha) \in H^{2 i p}(G, \mathbf{Z})$. (If $\alpha$ (of even degrees) is not homogeneous, then we denote by $\Re_{j}(\alpha)$, the component of $\Re(\alpha)$ of degree $2 j$. Often, the components $\Re_{j}(\alpha), 0<j<p$, can be expressed in terms of transfer.)

THEOREM III. Let $H$ be normal in $G$ of prime index $p$, and let $\xi: H \rightarrow U_{n}$ be a unitary representation. Let $\mu$ generate $H^{2}(G / H, Z)$, and let $\gamma=$ $\inf _{G / H \rightarrow G}(\mu)$. Then the Chern classes of the induced representation $\rho=$ ind $_{H \rightarrow G} \xi$ are given by

$$
\begin{aligned}
c(\rho)= & \Re(c(\xi))+\sum_{t=1}^{n-1} \Re\left(c_{t}(\xi)\right)\left[\left(1-\gamma^{p-1}\right)^{n-t}-1\right] \\
& +\left[\left(1-\gamma^{p-1}\right)^{n}-1\right] .
\end{aligned}
$$


Proof. We need only note that $\Phi^{*}(\mu)=\gamma$ where $\mu$ is identified with the appropriate element of $H^{2}\left(P \int H, Z\right)=H^{2}(P, Z) \oplus \ldots$

It is interesting to write out some of the components explicitly. (Some of these formulas have to be modified for special values of $p$ and $n$, e.g. $p=2$, $n=2$.)

$$
\begin{aligned}
c_{p n}(\rho) & =\Re_{p n}(c(\xi))=\Re\left(c_{n}(\xi)\right) \\
c_{p n-1}(\rho) & =\Re_{p n-1}(c(\xi))-\Re\left(c_{n-1}(\xi)\right) \gamma^{p-1} \\
c_{p n-2}(\rho) & =\Re_{p n-2}(c(\xi))+\Re\left(c_{n-2}(\xi)\right) \gamma^{2(p-1)}
\end{aligned}
$$

(Terms like $\Re_{p n-1}(c(\xi)), \Re_{p n-2}(c(\xi)), \ldots$ may be rewritten in terms of transfer $[H \rightarrow G]$, should it be necessary.)

$$
\begin{aligned}
& c_{p}(\rho)=\Re_{p}(c(\xi)) \\
& \left(\Re_{p}(c(\xi))=\Re\left(c_{1}(\xi)\right)+\text { transfers }\right) \\
& c_{p-1}(\rho)=\Re_{p-1}(c(\xi))-n \gamma^{p-1} \\
& c_{p-2}(\rho)=\Re_{p-2}(c(\xi)) \\
& \vdots \\
& c_{2}(\rho)=\Re_{2}(c(\xi)) \\
& c_{1}(\rho)=\Re_{1}(c(\xi))=\operatorname{tr}_{H \rightarrow G}\left(c_{1}(\xi)\right) .
\end{aligned}
$$

3. Some possible generalizations. The theorems of $\$ 2$ were not stated in the widest generality. Certain extensions immediately come to mind.

First, note that Theorem I could equally have been stated for $\S_{p} \int T$ by ignoring components relatively prime to $p$. As a result, in Theorem III, we could drop the assumption that $H$ is normal in $G$, but we would have to suppress components relatively prime to $p$.

To be even more general, it is possible to analyze the situation in $\mathcal{S}_{l} \int U_{n}$ where $l$ is not necessarily prime. As before, we have the diagram in Figure $1^{\prime}$. As before, $H^{*}\left(\delta_{l}, H^{*}\left(B U_{n}\right)^{l}\right) \rightarrow H^{*}\left(\delta_{l}, H^{*}\left(B T^{n}\right)^{l}\right)$ is a monomorphism. Hence, we may attempt to descend on the right using the results of [Ch] for $\delta_{l} \int T$. Unfortunately, we were unable to make much sense out of the calculations. About all that one can get easily are bounds on the orders of the components of the Chern classes additional to the elementary symmetric functions. (These terms involve Chern classes of the natural representations $\pi_{j}: \delta_{j} \rightarrow U_{j}$ for $0<j \leqslant n$ in a rather complicated way.) 


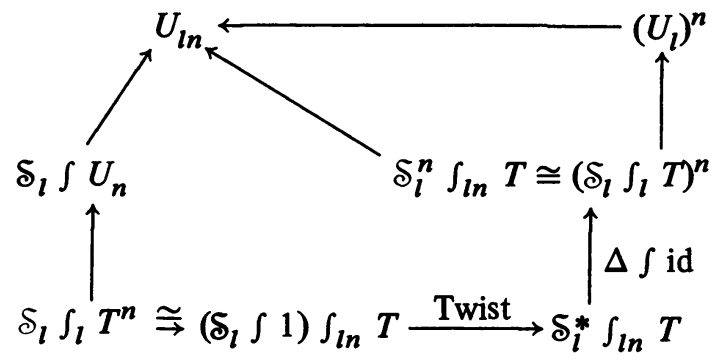

FiguRE $1^{\prime}$

4. Chern classes of natural representations of symmetric groups. We apply Theorem II of $\$ 2$ to the calculation of the Chern classes $c_{i}\left(\pi_{l}\right)$ of the natural representations $\pi_{l}: \delta_{l} \rightarrow U_{l}$ of symmetric groups by permutation matrices. Because of the known Sylow subgroup structure of symmetric groups [Ha, \$5.9, pp. 81-83], and because restriction to the p-Sylow subgroup is a monomorphism on the $p$-primary components [CE, Chapter XII, \&10, p. 259], it suffices to consider prime powers $l=p^{m}$.

Our conclusion is that the $p$-primary components of the integral Chern classes have orders exactly equal io the Grothendieck bounds ( $\$ 1)$ for $p$ odd but often one-half those bounds for $p=2$.

Before stating the theorem we need some notation. For a prime $p$, let $v_{p}$ denote the usual $p$-adic valuation in $\mathbf{Z}$, and if $A$ is an abelian group, denote

$$
\lambda_{p}(a)=\nu_{p}(\text { order of } a)
$$

for each $a \in A$.

THEOREM. Let $p$ be a prime. The integral Chern classes, $c_{i}\left(\pi_{p^{m}}\right) \in H^{2 i}\left(\delta_{p^{n}}\right.$, Z) have p-primary components given, for $p$ odd, by

$$
\lambda_{p}\left(c_{i}\left(\pi_{p^{m}}\right)\right)= \begin{cases}0 & \text { if } i \neq 0 \bmod p-1, \\ 1+\nu_{p}(i) & \text { if } i \equiv 0 \bmod p-1,\end{cases}
$$

$0<i<p^{m} ;$ and, for $p=2$, by

$$
\begin{gathered}
\lambda_{2}\left(c_{2^{m}}\left(\pi_{2^{m}}\right)\right)=0, \text { and } \\
\lambda_{2}\left(c_{i}\left(\pi_{2^{m}}\right)\right)=1+\nu_{2}(i),
\end{gathered}
$$

$0<i \leqslant 2^{m}-1$.

Proof. First, note that we may replace $\delta_{p^{m}}$ by its $p$-Sylow subgroup which we denote $P_{m}$. One knows that these Sylow subgroups may be constructed inductively as wreath products:

$$
P_{1}=P, \quad P_{m}=P \int P_{m-1} .
$$

Moreover, the natural representations of these subgroups are given by 


$$
\begin{aligned}
& \rho_{1}=\text { regular representation of } P_{1}=P, \\
& \rho_{m}=\text { id } \int \rho_{m-1}: P \int P_{m-1} \rightarrow U_{p^{m}} . \\
& \left(\rho_{m}=\text { restriction of } \pi_{p^{m}} \text { to } P_{m} .\right)
\end{aligned}
$$

Thus, the obvious way to proceed is by induction on $m$. (Note. For the case $p=2$, slight modifications in language will sometimes be necessary, but we mostly ignore this issue.)

For $m=1$,

$$
c\left(\rho_{1}\right)=1-\gamma^{p-1},
$$

so that the theorem is true.

Assume now $m>1$. We suppose inductively that $c\left(\rho_{m-1}\right)=c=1+c_{1}$ $+\cdots+c_{p^{m-1}}$ satisfies the conclusion of the theorem. For $\alpha \in H^{*}(H, Z)$ of even degrees, let $\omega_{i}(\alpha) \in H^{2 i}\left(P \int H, Z\right)$ be the homogeneous component of $1 \int \alpha$ of degree $2 i$.

We are now ready to use Theorem II of $\$ 2$ with $n=p^{m-1}, H=P_{m-1}$, $\xi=\rho_{m-1}$. Since $c_{i}=0$ for $i \neq 0 \bmod p-1$, we have also $\omega_{i}(c)=0$ for $i \neq 0$ $\bmod p-1$. In particular, $c_{p^{m-1}}=0$, and the nonzero component of $1 \int c$ of highest degree is $\omega_{p^{m}-p}(c)=1 \int c_{p^{m-1}-1}$. (We see below that this is nonzero.)

To clarify the situation we use the diagram in Figure 3.

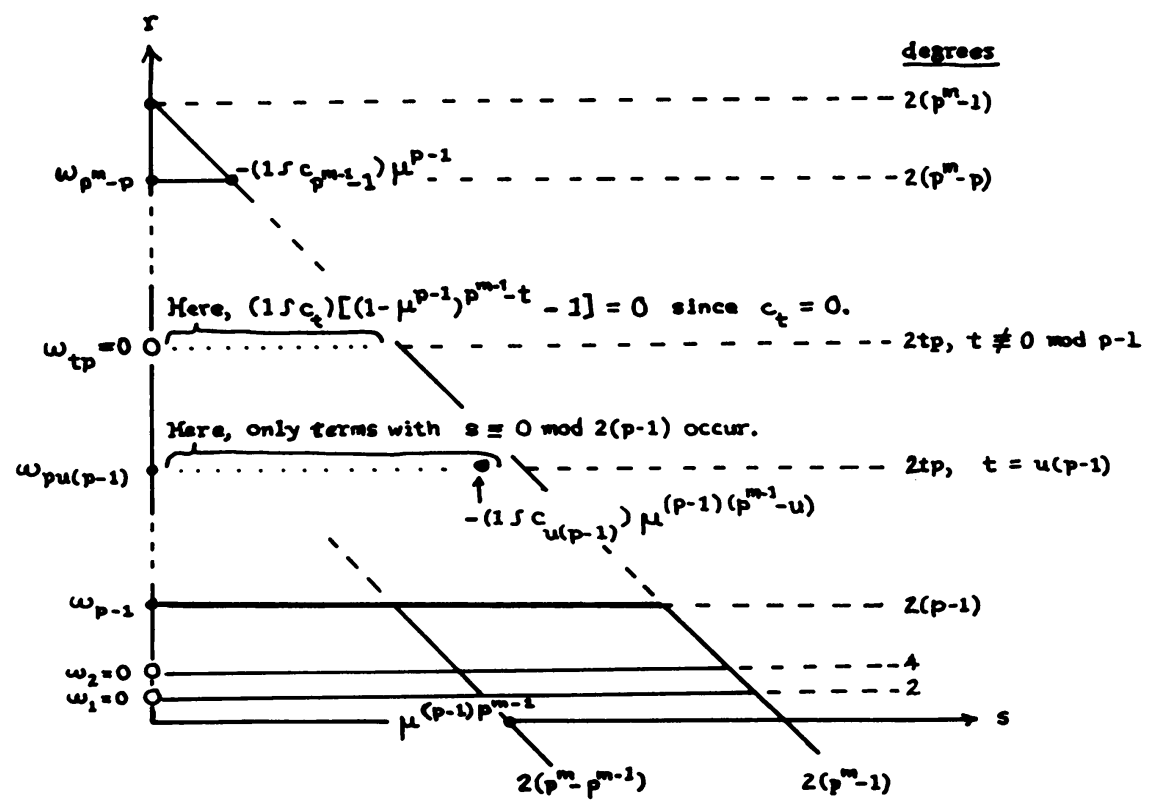

FigURE 3

The diagram roughly represents the spectral sequence of the group extension 
$1 \rightarrow H^{p} \rightarrow P \int H \rightarrow P \rightarrow 1$ except that since Nakaoka's Theorem fails, elements such as $\omega_{i}(c)$ or $1 \int c_{t}$ do not have any meaning in any terms of the spectral sequence. (Modulo the filtration, of course, they represent elements at the indicated positions.) One notes immediately that $c_{i}\left(\rho_{m}\right)=0$ for $i \neq 0$ $\bmod p-1$ since nonzero contributions either on the vertical edge or on the rows have degrees divisible by $p-1$. Also, the highest degree terms in the rows are

$$
\begin{gathered}
-\left(1 \int c_{p^{m-1}-1}\right) \mu^{p-1} \text { of } \mathrm{deg} / 2=p\left(p^{m-1}-1\right)+p-1=p^{m}-1 \\
-\left(1 \int c_{p^{m-1}-p}\right) \mu^{(p-1)\left[p^{m-1}-\left(p^{m-1}-p\right)\right]} \text { of } \mathrm{deg} / 2 \\
=p\left(p^{m-1}-p\right)+p(p-1)=p^{m}-p \\
\vdots \\
-\mu^{p-1\left[p^{m-1}\right]} \text { of } \mathrm{deg} / 2=p^{m}-p^{m-1} .
\end{gathered}
$$

Hence, the (potentially) nonzero Chern class of highest degree is

$$
c_{p^{m}-1}\left(\rho_{m}\right)=-\left(1 \int c_{p^{m-1}-1}\right) \mu^{p-1},
$$

and for $1<i<p^{m}-p, i \equiv 0 \bmod p-1$,

$$
c_{i}\left(\rho_{m}\right)=\omega_{i}(c)+\text { additional terms. }
$$

The "additional terms" are all of order at most $p$ (being multiples of $\mu^{p-1}$ ), and are in the kernel of res: $H^{*}\left(P \int P_{m-1}, Z\right) \rightarrow H^{*}\left(P_{m-1}^{p}, Z\right)$. We shall see that the order of $c_{i}\left(\rho_{m}\right), 0<i<p^{m}-p$, is determined by the order of the component $\omega_{i}(c)$.

LEMMA U. $\lambda_{p}\left(\omega_{i}(c)\right)<1+\nu_{p}(i)$.

Proof. We begin by analyzing $1 \int c=1 \int\left(1+c_{p-1}+c_{2(p-1)}+\cdots+\right.$ $\left.c_{p^{m-1}-1}\right)$ in greater detail. The homogeneous component of $\operatorname{deg} 2 i$ involves terms of two kinds:

(a) "cross-terms" which restrict in $\boldsymbol{P}_{\boldsymbol{m}-1}^{p}$ to terms of the form

$$
\begin{aligned}
c_{i_{1}} \times c_{i_{2}} \times \cdots \times c_{i_{p}}+c_{i_{2}} \times c_{i_{3}} & \times \cdots \times c_{i_{1}} \\
& +\cdots+c_{i_{p}} \times c_{i_{1}} \times \cdots \times c_{i_{p-1}},
\end{aligned}
$$

where

$$
i_{1}+i_{2}+\cdots+i_{p}=i(\text { all } \equiv 0 \bmod p-1),
$$

and the indices are not all the same, or, if $i \equiv 0 \bmod p$,

(b) one term of the form $1 \int c_{j}(p j=i)$, which restricts in $P_{m-1}^{p}$ to $c_{j} \times c_{j} \times \cdots \times c_{j}$.

To see this, choose a cocycle $f=f_{0}+f_{p-1}+\cdots+f_{p^{m-1}-1}$ representing $c$, and expand the cocycle $\varepsilon \otimes f^{p}=\varepsilon \int f$ representing $1 \int c$. See $[\mathbf{N}, \S 4$, item 5 , 
p. 58]. Also, note that the cocycle representation shows that each type (a) term is the transfer $\left[P_{m-1}^{p} \rightarrow P_{m}\right]$ of the element $c_{i_{1}} \times c_{i_{2}} \times \cdots \times c_{i_{p}} \in$ $H^{2 i}\left(P_{m-1}^{p}, \mathbf{Z}\right)$. (See [N, pp. 63-64] for an analogous argument.)

Suppose first that $i \equiv 0 \bmod p-1$, but $i \neq 0 \bmod p$. Then, no type (b) term occurs, and, for each type (a) term, one of the indices $i \neq 0 \bmod p$. In that case, $p\left(c_{i_{1}} \times \cdots \times c_{i} \times \cdots \times c_{i_{p}}\right)=0$, and the same is true for the transfer of this element. Hence $\omega_{i}(c)$ is a sum of terms, each of order at most p.

Suppose next that $i=j p$ where $j \equiv 0 \bmod p-1$. For $i=i_{1}+i_{2}+\cdots+$ $i_{p}$,

$$
\nu_{p}(i) \geqslant \min _{i_{u} \neq 0} \nu_{p}\left(i_{u}\right) .
$$

Hence, for $i_{1}, i_{2}, \ldots, i_{p} \equiv 0 \bmod p-1$,

$$
\lambda_{p}\left(c_{i_{1}} \times c_{i_{2}} \times \cdots \times c_{i_{p}}\right)=\min _{i_{u} \neq 0} \nu_{p}\left(i_{u}\right)+1 \leqslant \nu_{p}(i)+1,
$$

and therefore the same is true for type (a) terms. Thus, to complete the proof of the lemma, we need to show

$$
\begin{aligned}
\lambda_{p}\left(1 \int c_{j}\right) & \leqslant 1+\nu_{p}(i)=1+\nu_{p}(j)+1 \quad \text { (by induction) } \\
& =\lambda_{p}\left(c_{j}\right)+1 .
\end{aligned}
$$

This follows from the following calculation. Let $q=p^{\lambda_{p}\left(c_{j}\right)}$. Then

$$
\begin{aligned}
q(\operatorname{tr} \cdot \operatorname{res})\left(1 \int c_{j}\right) & =q \operatorname{tr}\left(c_{j} \times c_{j} \times \cdots \times c_{j}\right) \\
& =\operatorname{tr}\left(q\left(c_{j} \times \cdots \times c_{j}\right)\right)=0 .
\end{aligned}
$$

On the other hand,

$$
\operatorname{tr} \cdot \operatorname{res}\left(1 \int c_{j}\right)=\left(P_{m}: P_{m-1}\right)\left(1 \int c_{j}\right)=p\left(1 \int c_{j}\right) .
$$

Hence, $q p\left(1 \int c_{j}\right)=0$ as required.

The lemma proved, we now conclude

$$
\lambda_{p}\left(c_{i}\left(\rho_{m}\right)\right)<\nu_{p}(i)+1
$$

for $i \equiv 0 \bmod p-1, i>0$. For, if $0<i \leqslant p^{m}-p$, the additional terms have order at most $p$, and if $i=p^{m}-1, c_{i}\left(\rho_{m}\right)=-\left(1 \int c_{p^{m-1}-1}\right) \mu^{p-1}$ has order at most $p$ as claimed.

LEMMA L. $\lambda_{p}\left(\omega_{i}(c)\right) \geqslant 1+\nu_{p}(i)$.

Proof. Suppose first that $i \equiv 0 \bmod p-1$, but $i \neq 0 \bmod p$. Then, as above, no type (b) term occurs, and, each type (a) term restricts to something of the form

$$
c_{i_{1}} \times c_{i_{2}} \times \cdots \times c_{i_{p}}+c_{i_{2}} \times \cdots \times c_{i_{1}}+\cdots+c_{i_{p}} \times \cdots \times c_{i_{p-1}},
$$

where $i=i_{1}+\cdots+i_{p}\left(\right.$ each $\left.i_{u} \equiv 0 \bmod p-1\right)$, the indices are not all the 
same, and also at least one $i_{u} \not \neq 0 \bmod p$. The latter expression is exactly of order $p$. For, each term in the sum is exactly of order $p$. Also, the terms belong to different direct summands in the Künneth Theorem decomposition of $H^{2 i}\left(P_{m-1}^{p}, \mathrm{Z}\right)$, so they cannot add up to zero. It follows that each type (a) term restricts to something of order $p$, and by the same Künneth Theorem argument, their sum $\omega_{i}(c)$ restricts to something of order $p$, and hence $\lambda_{p}\left(\omega_{i}(c)\right) \geqslant 1$.

Suppose next that $i \equiv 0 \bmod p(p-1)$; let $i=j p$ with $j \equiv 0 \bmod p-1$. Consider first the type (a) term $\operatorname{tr}\left(c_{i_{1}} \times c_{i_{2}} \times \cdots \times c_{i_{p}}\right)$. The argument in Lemma $\mathrm{U}$ shows that $\nu_{p}(\operatorname{tr}(-))$ is bounded above by $\min _{i_{u} \neq 0} \nu_{p}\left(i_{u}\right)+1$, and the argument presented above (restricting to $P_{m-1}^{p}$ ) shows that its order is bounded below by the same quantity; hence this is its exact order. Suppose one of these type (a) terms has order $p^{p_{p}(i)+1}$. Then it is easy to see $\lambda_{p}\left(\omega_{i}(c)\right) \geqslant \nu_{p}(i)+1$. For, $\operatorname{res}\left(p^{\nu_{p}(i)} \omega_{i}(c)\right)$ is a sum of terms of the form (type (a))

$$
p^{p_{p}(i)}\left(c_{i_{1}} \times c_{i_{2}} \times \cdots \times c_{i_{p}}+\cdots+c_{i_{p}} \times c_{i_{1}} \times \cdots \times c_{i_{-1}}\right)
$$

plus one additional (type (b)) term

$$
p^{\nu_{p}(i)}\left(c_{j} \times c_{j} \times \cdots \times c_{j}\right) .
$$

Since $\nu_{p}(i)=\nu_{p}(j)+1$, the latter is zero, and we have assumed at least one of the former terms is nonzero. By the Künneth argument used above, $\operatorname{res}\left(p^{\nu_{p}(i)} \omega_{i}(c)\right) \neq 0$. Suppose, then, on the other hand, that every type (a) term has order $\leqslant p^{v_{p}(i)}$ (as would certainly be true for $i>p^{m-1}$, for example). Then, to prove the lemma, it suffices to prove $\lambda_{p}\left(1 \int c_{j}\right) \geqslant \nu_{p}(i)+1$. We shall prove this below. (Subcomplex argument.)

Given Lemmas $U$ and $\mathrm{L}$, we can (almost) complete the proof of the theorem. If $\lambda_{p}\left(\omega_{i}(c)\right)>1, \lambda_{p}\left(c_{i}\left(\rho_{m}\right)\right)=\lambda_{p}\left(\omega_{i}(c)\right)$ since the additional terms (to be added to $\left.\omega_{i}(c)\right)$ have order at most $p$. If $\lambda_{p}\left(\omega_{i}(c)\right)=1$, we need only show $c_{i}\left(\rho_{m}\right) \neq 0$ (because then its order is necessarily $\left.p\right)$. But, since the additional terms are in $\operatorname{Ker}(\operatorname{res}), \operatorname{res}\left(c_{i}\left(\rho_{m}\right)\right)=\operatorname{res}\left(\omega_{i}(c)\right) \neq 0$ as above. Thus, we can conclude finally that

$$
\lambda_{p}\left(c_{i}\left(\rho_{m}\right)\right)=\nu_{p}(i)+1,
$$

$0<i \leqslant p^{m}-p$. Thus, it remains only to show that $\left(1 \int c_{p^{m-1}-1}\right) \mu^{p-1} \neq 0$ (so that its order is necessarily $p$ ). One can see this directly by a simple analysis of the product structure in the $E_{2}$-term of the spectral sequence of the group extension $1 \rightarrow P_{m-1}^{p} \rightarrow P_{m} \rightarrow P \rightarrow 1$. However, it also follows from the subcomplex argument presented below.

Subcomplex argument. Let $C$ be a cochain complex which is Z-free and suppose $\beta \in H^{2 i}(C, Z)$. Let $q$ be a power of the prime $p$. Suppose there is $a$ homomorphism $\psi: H^{2 i}(C, \mathbf{Z}) \rightarrow \mathbf{Z} / q \mathbf{Z}$ such that $\psi(q)$ generates $\mathbf{Z} / q \mathbf{Z}$. Let $W$ 
be a $P$-free resolution of $\mathbf{Z}$. Then, we claim that

$$
1 \int \beta \in H^{2 i p}\left(\operatorname{Hom}_{P}\left(W, C^{\otimes_{p}}\right)\right)
$$

has order $>p q$. Also, $\left(1 \int \beta\right) \mu^{l} \neq 0$ in $H^{2 i p+2 l}\left(\operatorname{Hom}_{P}\left(W, C^{\otimes p}\right)\right)$ for $l>0$. The product arises from the obvious pairing

$$
\underbrace{H^{*}\left(\operatorname{Hom}_{P}\left(W, \mathbf{Z}^{\otimes p}\right)\right) \otimes}_{\begin{array}{c}
\text { IIl } \\
H^{*}(P, \mathbf{Z}) .
\end{array}} H^{*}\left(\operatorname{Hom}_{P}\left(W, C^{\otimes p}\right)\right) \rightarrow H^{*}\left(\operatorname{Hom}_{P}\left(W, C^{\otimes p}\right)\right)
$$

In our application, $C=\operatorname{Hom}_{P_{m-1}}(X, \mathbf{Z})$, where $X$ is a $P_{m-1}$-resolution of $\mathbf{Z}$, $\beta=c_{j}$, and $q$ is the order of $c_{j}$. (We shall show the existence of the required homomorphism $\psi$ inductively later.)

Proof of Claims. (Note: The use of the module $M$ in the following argument was suggested by the analysis in [ST].)

Let $\beta \in H^{2 i}(C), \psi: H^{2 i}(C) \rightarrow \mathrm{Z} / q \mathrm{Z}$ be as above. For convenience, write $n=2 i$. Let $Z^{n}$ and $B^{n}$ denote the cocycles and coboundaries of the complex $C$. Form the usual $Z$-free cochain complex $M$ as follows:

$$
\begin{gathered}
M^{n-1}=\mathbf{Z} y, \quad M^{n}=\mathbf{Z} x, \quad M^{j}=0 \text { otherwise; } \\
d^{n-1}(y)=q x .
\end{gathered}
$$

Then $H^{j}(M)=0$ for $j \neq n$, and $H^{n}(M)=\mathbf{Z} / q \mathrm{Z}$. Moreover, because $Z^{n}$ is free, we can fill in the diagram

$$
\begin{array}{ccccccccc}
0 & \rightarrow & B^{n} & \rightarrow & Z^{n} & \rightarrow & H^{n}(C) & \rightarrow & 0 \\
& & \psi \downarrow & & \psi \downarrow & & \psi \downarrow & & \\
0 & \rightarrow & M^{n-1} & \rightarrow & M^{n} & \rightarrow & \mathrm{Z} / q \mathrm{Z} & \rightarrow & 0 .
\end{array}
$$

(Call all the vertical maps $\psi$ for convenience.) Finally, as $C^{n}=B^{n+1} \oplus Z^{n}$, we can extend $\psi$ to a map of complexes

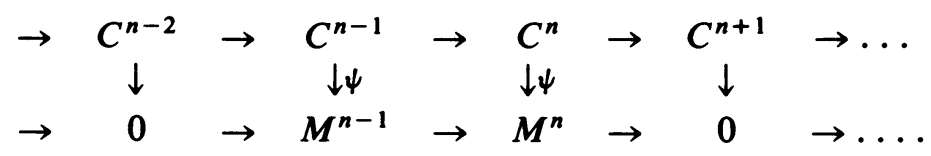

Because of the naturality of the wreath product under $\operatorname{Hom}_{P}\left(\mathrm{id}, \psi^{\otimes_{p}}\right)$ it suffices to analyze $1 \int \psi(\beta) \in H^{n p}\left(\operatorname{Hom}_{P}\left(W, M^{\otimes_{p}}\right)\right)$. Since $\psi(\beta) \in H^{n}(M)$ $\cong \mathrm{Z} / q \mathrm{Z}$ generates, we have $\psi(\beta)=t \chi$ where $\chi \leftrightarrow 1$ and $(t, q)=1$. On the other hand, it is easy to see that $H^{*}\left(\operatorname{Hom}_{P}\left(W, M^{\otimes p}\right)\right)$ is a $p$-group. (For, by the Künneth Theorem, $H^{*}\left(M^{\otimes p}, \mathrm{Z}\right)$ is a $p$-group, and hence, so is the $E_{2}$-term of the "first" spectral sequence

$$
H^{*}\left(P, H^{*}\left(M^{\otimes p}, \mathrm{Z}\right)\right) \Rightarrow H^{*}\left(\operatorname{Hom}_{P}\left(W, M^{\otimes p}\right)\right)
$$

of the double complex.) Thus, we may replace $1 \int \psi(\beta)=1 \int t \chi=t^{p}\left(1 \int \chi\right)$ 
by $1 \int \chi$ in our deliberations. Notice that since $x \in M^{n}$ represents $\chi, \varepsilon \int x \in$ $\operatorname{Hom}_{P}\left(W_{0},\left(M^{n}\right)^{\otimes_{p}}\right)$ represents $1 \int \chi .\left(\varepsilon \int x\right.$ is defined in [N, p. 58]. If we choose $W$ with $W_{0}=\mathbf{Z}[P]$, we may identify $\varepsilon \int x$ with $x \otimes x \otimes \cdots \otimes x \in$ $\left(M^{n}\right)^{\otimes p}$.)

To calculate in the double complex $\operatorname{Hom}_{P}\left(W, M^{\otimes p}\right)$, we consider the so-called "second" spectral sequence, [CE, Chapter XV, §6, pp. 330-333]

$$
H^{*}\left(H^{*}\left(P, M^{\otimes p}\right)\right) \Rightarrow H^{*}\left(\operatorname{Hom}_{P}\left(W, M^{\otimes p}\right)\right) .
$$

To calculate $H^{*}(P, M \otimes M \otimes \cdots \otimes M)$, we must first analyze $M^{\otimes p}$ as a $P$-module. The case $p=2$ is a little different, so we suppose $p>2$. Then, $M^{\otimes p}$ breaks up as follows into homogeneous components:

$$
\begin{aligned}
& \text { degree } \quad \underline{\text {-basis }} \quad \underline{P \text {-module }} \\
& n p \quad-x \otimes x \otimes \cdots \otimes x-\mathbf{Z} \\
& n p-1-y \otimes x \otimes \cdots \otimes x, \quad-\mathrm{Z}[P] \\
& x \otimes x \otimes \cdots \otimes y, \\
& x \otimes y \otimes \cdots \otimes x \\
& n p-2-\longrightarrow-\frac{p-1}{2} \text { copies of } \mathbf{Z}[P] \\
& n p-p+1-x \otimes y \otimes \cdots \otimes y, \quad-\quad Z[P] \\
& y \otimes y \otimes \cdots \otimes x, \\
& -y \otimes \cdots \otimes y \otimes x \otimes y, \\
& -y \otimes x \otimes y \otimes \cdots \otimes y \\
& n p-p-y \otimes y \otimes \cdots \otimes y \quad-\mathbf{Z}
\end{aligned}
$$

In particular, except in the extreme degrees $n p$ and $n p-p$, the module $\left(M^{\otimes p}\right)^{j}$, of degree $j$, is free over $\mathbf{Z}[P]$. Hence, $H^{0}\left(P,\left(M^{\otimes p}\right)^{j}\right)$ is $\mathbf{Z}$-free and $H^{r}\left(P,\left(M^{\otimes p}\right)^{j}\right)=0$ for $r>0$. Thus, the first term of the spectral sequence, $\tilde{E}$, can be represented diagrammatically: 


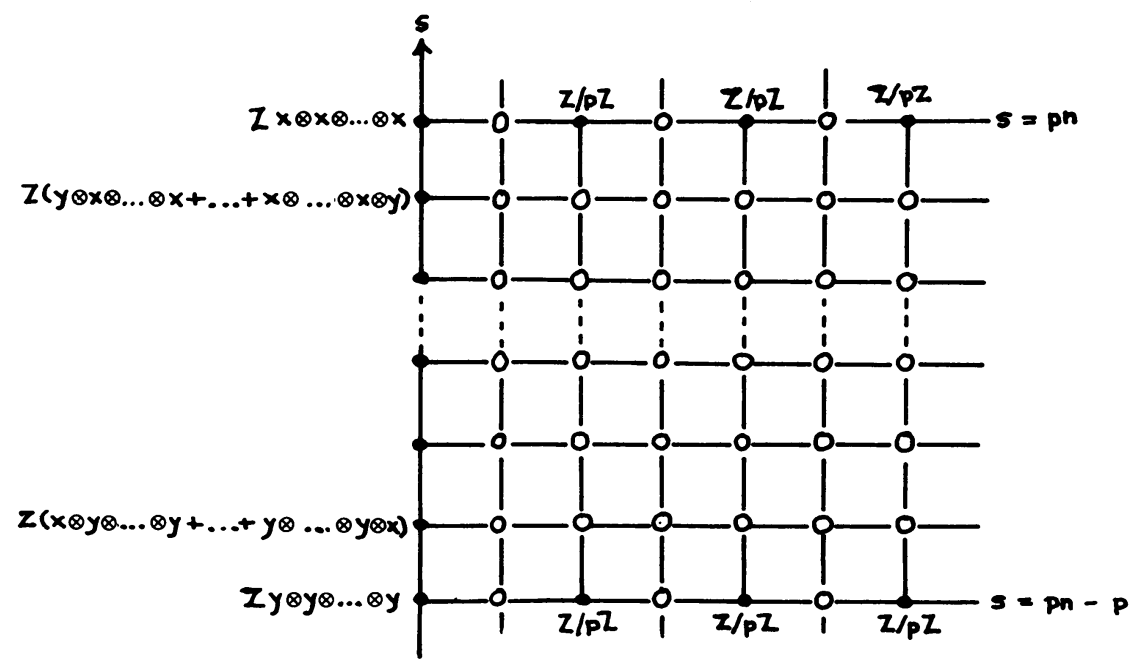

(Note that $y \otimes \cdots \otimes y$ is in fact invariant under $\sigma$ (the generator of $P$ ) despite the signs involved in the action of $P$ on the complex. For, $p$ being odd, $\sigma$ is an even permutation.) The differential $\tilde{d}_{1}$ is trivial except on the vertical edge. There, $\tilde{d}_{1}^{0, p n-1}$, the relevant differential, is calculated as follows:

$$
\begin{aligned}
d(y \otimes x \otimes \cdots \otimes x+x \otimes x \otimes \cdots \otimes y+x \otimes \cdots \otimes y \otimes x \\
\quad+\cdots+x \otimes y \otimes \cdots \otimes x) \\
=q x \otimes \cdots \otimes x+q x \otimes \cdots \otimes x+\cdots+q x \otimes \cdots \otimes x \\
=q p(x \otimes x \otimes \cdots \otimes x) .
\end{aligned}
$$

(Similarly, at the lower end, $\tilde{d}_{1}^{0, n p-p}: \mathbf{Z} \rightarrow \mathbf{Z}$ is multiplication by $q$.) Thus, the $\tilde{E}_{2}$-term of the spectral sequence looks like the following:

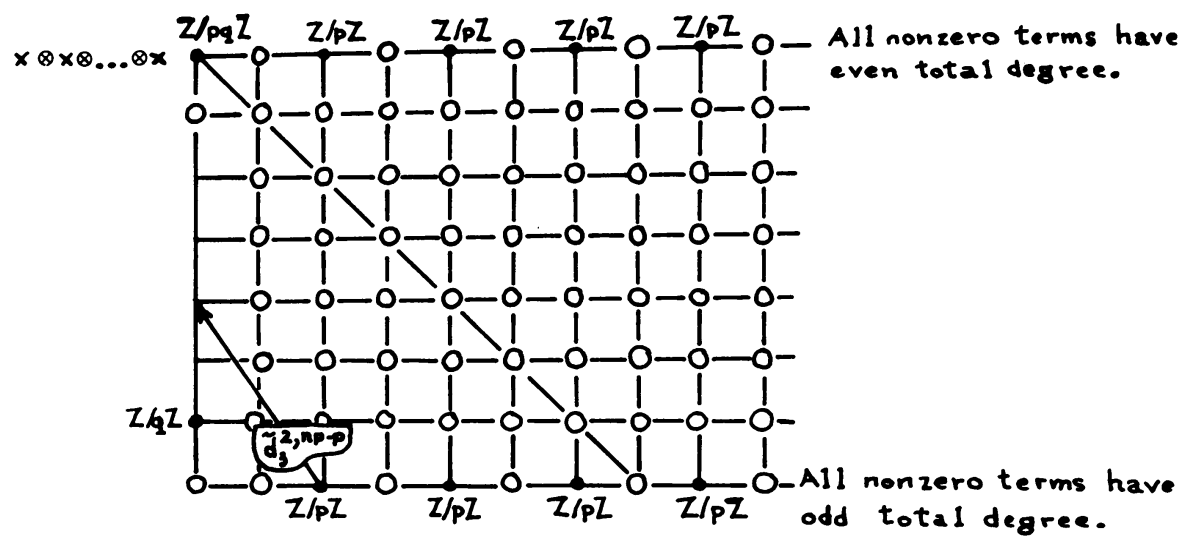

FIGURE 4 
We claim that $\tilde{d}_{r}=0$ for $r>0$. To see this, it suffices to calculate the differentials for $s=n p-p$, (the bottom edge). Moreover, it suffices to show

$$
\tilde{d}_{3}^{2, n p-p}: H^{n p-p}\left(H^{2}\left(P, M^{\otimes p}\right)\right) \rightarrow H^{n p-p+1}\left(H^{0}\left(P, M^{\otimes p}\right)\right)
$$

is trivial. For, viewing the entire complex as a module over $\operatorname{Hom}_{P}\left(W, \mathbf{Z}^{\otimes_{p}}\right)$ (for which the spectral sequence trivially reduces to $H^{*}(P, \mathrm{Z})$ ), we note that every generator on the aforementioned bottom edge may be obtained by multiplying a generator in position $(n p-p, 2)$ by some power of $\mu \in$ $H^{2}(P, \mathrm{Z})$.

To show $\tilde{d}_{3}^{2, n p-p}=0$, we calculate the order of $H^{p n-p+2}\left(\operatorname{Hom}_{P}\left(W, M^{\otimes p}\right)\right)$. To do this, it will be easier to work in homology. Let $M=\operatorname{Hom}_{\mathrm{z}}(N, Z)$, where $N_{n-1}=\mathrm{Zu}, N_{n}=\mathrm{Z} v$ and $d_{n}(v)=q u$. Then

$$
\operatorname{Hom}_{P}\left(W, M^{\otimes_{p}}\right) \cong \operatorname{Hom}_{\mathbf{Z}}\left(W \otimes_{P} N^{\otimes_{p}}, \mathbf{Z}\right)
$$

and

$$
H^{0}\left(P, M^{\otimes p}\right) \cong \operatorname{Hom}_{\mathrm{z}}\left(H_{0}\left(P, N^{\otimes_{p}}\right), \mathrm{Z}\right) .
$$

(Thus, we may use the universal coefficient theorem to calculate the cohomology on the left.)

The $P$-module structure of $N^{\otimes p}$ in the relevant lower degrees is indicated in the table:

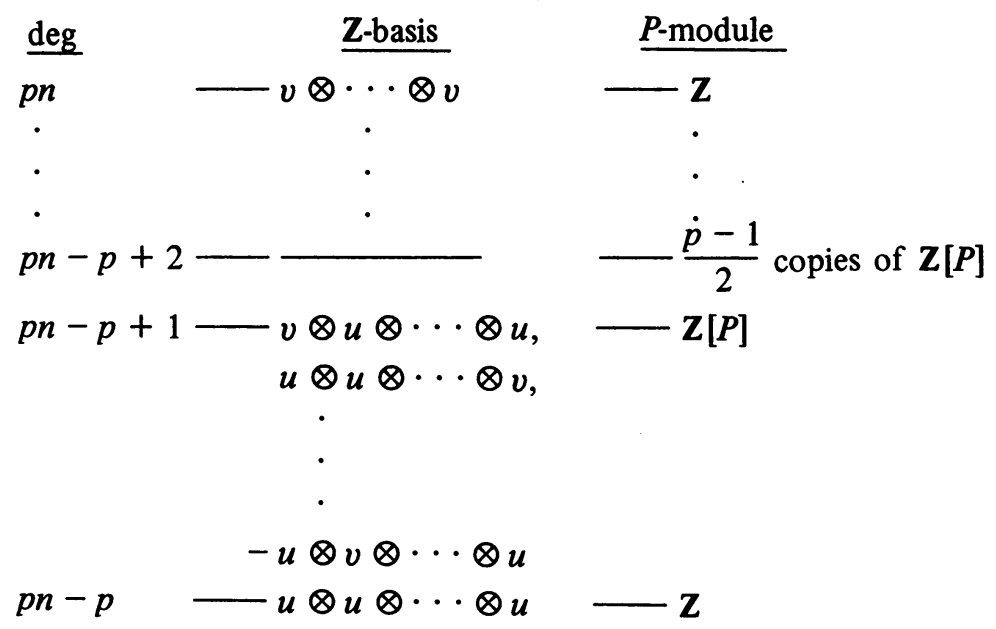

The $\tilde{E}^{1}$-term is similar to what occurred above, but at the lower end, we have

$$
\tilde{d}_{0, p n-p+1}^{1}: H_{0}\left(P,\left(N^{\otimes p}\right)_{p n-p+1}\right) \cong \mathrm{Z} \rightarrow H_{0}\left(P,\left(N^{\otimes p}\right)_{n p-p}\right) \cong \mathrm{Z}
$$

which is multiplication by $q$. [Modulo $\sigma-1, v \otimes u \otimes \cdots \otimes u$ generates, and $d(v \otimes u \otimes \cdots \otimes u)=q(u \otimes \cdots \otimes u)$.] Thus 


$$
\begin{aligned}
H_{p n-p+1}\left(H_{0}\left(P, N^{\otimes p}\right)\right) & =0, \\
H_{p n-p}\left(H_{0}\left(P, N^{\otimes p}\right)\right) & =\mathrm{Z} / q \mathrm{Z} .
\end{aligned}
$$

The $\tilde{E}^{2}$-term of the spectral sequence looks like

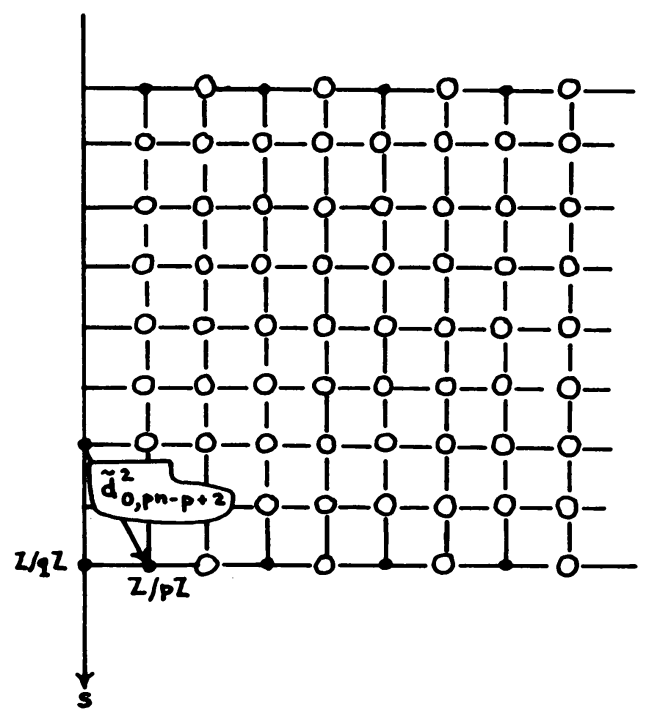

Moreover, $\tilde{d}_{0, p n-p+2}^{2}=0$ (in fact $\tilde{d}^{r}=0$ for $r>0$ ). For, we may tensor with $\mathbf{Z} / p \mathbf{Z}$ to get a complex $\bar{N}$ with vertical differential $d_{n}: \mathbf{Z} / p \mathbf{Z} \stackrel{q}{\rightarrow} \mathbf{Z} / p \mathbf{Z}$ trivial. In the spectral sequence for $W \otimes_{P} \bar{N}^{\otimes p}$ all differentials $\tilde{d}^{r}=0, r>1$. Comparing the spectral sequences and noting that

$$
H_{n p-p}\left(H_{1}\left(P, N^{\otimes p}\right)\right)=\mathrm{Z} / p \mathrm{Z} \rightarrow H_{n p-p}\left(H_{1}\left(P, \bar{N}^{\otimes p}\right)\right)=\mathrm{Z} / p \mathrm{Z}
$$

is an isomorphism allows us to conclude that $\tilde{d}_{0, p n-p+2}^{2}=0$. Thus,

$$
H_{p n-p+1}\left(W \otimes_{P} N^{\otimes_{p}}\right) \cong \mathrm{Z} / p \mathrm{Z}
$$

The universal coefficient theorem for cohomology now yields

$$
H^{p n-p+2}\left(H^{0}\left(P, M^{\otimes p}\right)\right) \cong \operatorname{Ext}\left(H_{p n-p+1}\left(H_{0}\left(P, N^{\otimes p}\right)\right), \mathrm{Z}\right)=0
$$

(using (H1)), and

$$
\begin{aligned}
H^{p n-p+2}\left(\operatorname{Hom}_{P}\left(W, M^{\otimes p}\right)\right) & \cong \operatorname{Ext}\left(H_{p n-p+1}\left(W \otimes_{P} M^{\otimes_{p}}\right), \mathrm{Z}\right) \\
& \cong \mathrm{Z} / p \mathrm{Z}
\end{aligned}
$$

(using (H2)). It follows that $\tilde{d}_{3}^{2, n p-p}=0$, since otherwise, using (C1), we conclude that the left-hand side of (C2) is trivial.

The basic contentions are now proved (at least for $p$ odd). For examination of Figure 4 yields

$$
H^{p n}\left(\operatorname{Hom}_{P}\left(W, M^{\otimes p}\right)\right) \approx \mathbf{Z} / p q \mathrm{Z}
$$


with generator $1 \int \chi$ (represented by $x \otimes x \otimes \cdots \otimes x$ ), and

$$
H^{p n+2(p-1)}\left(\operatorname{Hom}_{P}\left(W, M^{\otimes p}\right)\right) \cong \mathbf{Z} / p \mathrm{Z}
$$

with generator $\left(1 \int \chi\right) \mu^{p-1}$.

The case $p=2$ is quite similar but differs in details. $\sigma$ does not act trivially on $\left(M_{n-1}\right)^{\otimes 2}=\mathbf{Z} y \otimes y$; instead $\sigma(y \otimes y)=-y \otimes y$.

The $\tilde{E}_{2}$-term of the spectral sequence looks like

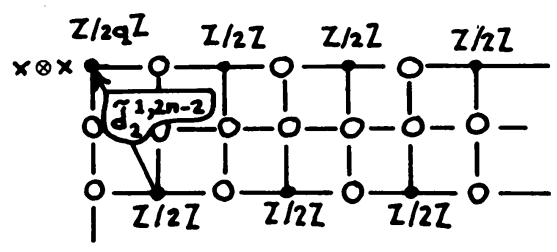

One can show $\tilde{d}_{2}^{1,2 n-2}=0$ fairly easily by direct calculation in the complex or one can use a duality argument as above.

Note. The method we have employed reduces the study of the homology of $W \otimes_{P} N^{\otimes_{p}}$ to that of $H_{0}\left(P, N^{\otimes_{p}}\right)$. This is an interesting $\mathrm{Z}$-free chain complex, and the method is perhaps interesting as a direct method for studying integral homology. Note in particular that the "increase in torsion" for $1 \int \chi$ is immediately apparent.

With the completion of the subcomplex argument, we are ready to finish the proof of the theorem. To do so, we need to define appropriate homomorphisms $\psi$. For $m=1, c_{p-1}\left(\rho_{1}\right)=-\mu^{p-1}$ generates $H^{2(p-1)}\left(P_{1}, Z\right)$ which is cyclic of order $p$. Suppose inductively we have shown that $c_{i}\left(\rho_{m}\right)$ is of order $q^{\prime}=p^{\nu_{p}(i)+1}(i \equiv 0 \bmod p-1)$. To continue the induction, we need $\psi^{\prime}$ : $H^{2 i}\left(P_{m}, \mathbf{Z}\right) \rightarrow \mathbf{Z} / q^{\prime} \mathbf{Z}$ such that $\psi^{\prime}\left(c_{i}\left(\rho_{m}\right)\right)$ generates. Suppose first that $0<i$ $<p^{m}-p$. If there is a type (a) term

$$
\operatorname{tr}\left(c_{i_{1}} \times \cdots \times c_{i_{p}}\right)
$$

of order $q^{\prime}$ in $\omega_{i}(c)$, the restriction of this term to $H^{2 i}\left(P_{m-1}^{p}, Z\right)$ is a sum of terms of order $q^{\prime}$. Using the Künneth Theorem, we can obtain a homomorphism onto $\mathbf{Z} / q^{\prime} \mathbf{Z}$ such that res $\left(\omega_{i}(c)\right)$ goes into a generator. Since res $\left(c_{i}\left(\rho_{m}\right)\right)$ $=\operatorname{res}\left(\omega_{i}(c)\right)$, one sees how to obtain the desired $\psi^{\prime}$. If, on the other hand, $i=j p$ and every type (a) term in $\omega_{i}(c)$ is of order $\left\langle q^{\prime}\right.$, then $1 \int c_{j}$ is of order $q^{\prime}$. Inductively, $\psi: H^{2 j}\left(P_{m-1}, \mathbf{Z}\right) \rightarrow \mathbf{Z} / q \mathrm{Z}\left(q^{\prime}=p q\right)$ carries $c_{j}$ to a generator of Z/qZ. As above, in the subcomplex argument, $\operatorname{Hom}_{P}\left(\mathrm{id}, \psi^{\otimes p}\right)$ carries $1 \int c_{j}$ to a generator of $H^{2 i p}\left(\operatorname{Hom}_{P}\left(W, M^{\otimes p}\right)\right)=\mathbf{Z} / p q \mathbf{Z}=\mathbf{Z} / q^{\prime} \mathbf{Z}$. Since $\psi$ carries every other component of $c\left(\rho_{m-1}\right)$ to 0 (by degree considerations), it follows that $\operatorname{Hom}_{P}\left(\mathrm{id}, \psi^{\otimes_{p}}\right)$ is trivial on every term contributing to $c_{i}\left(\rho_{m}\right)$ except $1 \int c_{j}$. Thus, $\operatorname{Hom}_{P}\left(\mathrm{id}, \psi^{\otimes p}\right)$ induces the required $\psi^{\prime}$. Finally, for $i=p^{m}-1$, 
$c_{i}\left(\rho_{m}\right)=-\left(1 \int c_{p^{m-1}-1}\right) \mu^{p-1}$, and, as above, $\operatorname{Hom}_{P}\left(\mathrm{id}, \psi^{\otimes p}\right)$ induces the required $\psi^{\prime}$.

Note. It is in fact true that, at each stage, $H^{*}\left(\operatorname{Hom}_{P}\left(W, M^{\otimes p}\right)\right)$ is a direct summand of $H^{*}\left(P_{m}, Z\right)$, and $c_{i}\left(\rho_{m}\right)$ generates a direct summand of $H^{2 i}\left(P_{m}\right.$, $Z$ ), but it was possible to get away with a little less in the proof.

\section{REFERENCES}

[CH] L. Evens, On the Chern classes of representations of finite groups, Trans. Amer. Math. Soc. 115 (1965), 180-193.

[N] $\longrightarrow$ A generalization of the transfer map in the cohomology of groups, Trans. Amer. Math. Soc. 108 (1963), 54-65.

[Bo] A. Borel, Topics in the homology theory of fibre bundlees, Lecture Notes in Math., no. 36, Springer-Verlag, New York, 1967.

[Ha] M. Hall, The theory of groups, Macmillan, New York, 1959.

[ST] N. E. Steenrod and E. Thomas, Cohomology operations derived from cyclic groups, Comment. Math. Helv. 32 (1957), 129-152.

[Th] C. B. Thomas, An integral Riemann-Roch formula for flat line bundles, Proc. London Math. Soc. 34 (1977), 87-101.

[G] A. Grothendieck, Classes de Chern et représentations linéaires des groupes discrets, in Dix exposés sur la cohomologie des schémas, Advanced Studies in Pure Mathematics, vol. 3, NorthHolland, Amsterdam, 1968.

[CE] H. Cartan and S. Eilenberg, Homological algebra, Princeton Univ. Press, Princeton, N.J., 1956.

Department of Mathematics, Northwestern Universtty, Evanston, Illinois 60201 